\title{
Suspect and nontarget screening approaches to identify organic contaminant records in lake sediments
}

\author{
Journal Article \\ Author(s): \\ Chiaia-Hernández, Aurea C.; Schymanski, Emma L.; Kumar, Praveen; Singer, Heinz P.; Hollender, Juliane \\ Publication date: \\ 2014 \\ Permanent link: \\ https://doi.org/10.3929/ethz-b-000091309
}

Rights / license:

In Copyright - Non-Commercial Use Permitted

Originally published in:

Analytical and Bioanalytical Chemistry 406(28), https://doi.org/10.1007/s00216-014-8166-0 


\title{
Suspect and nontarget screening approaches to identify organic contaminant records in lake sediments
}

\author{
Aurea C. Chiaia-Hernandez • Emma L. Schymanski • \\ Praveen Kumar • Heinz P. Singer • Juliane Hollender
}

Received: 10 June 2014 / Revised: 30 August 2014 / Accepted: 4 September 2014 / Published online: 26 September 2014

(C) Springer-Verlag Berlin Heidelberg 2014

\begin{abstract}
Sediment cores provide a valuable record of historical contamination, but so far, new analytical techniques such as high-resolution mass spectrometry (HRMS) have not yet been applied to extend target screening to the detection of unknown contaminants for this complex matrix. Here, a combination of target, suspect, and nontarget screening using liquid chromatography (LC)-HRMS/MS was performed on extracts from sediment cores obtained from Lake Greifensee and Lake Lugano located in the north and south of Switzerland, respectively. A suspect list was compiled from consumption data and refined using the expected method coverage and a combination of automated and manual filters on the resulting measured data. Nontarget identification efforts were focused on masses with $\mathrm{Cl}$ and $\mathrm{Br}$ isotope information available that exhibited mass defects outside the sample matrix, to reduce the effect of analytical interferences. In silico methods combining the software MOLGEN-MS/MS and MetFrag were used for direct elucidation, with additional consideration of retention
\end{abstract}

Electronic supplementary material The online version of this article (doi:10.1007/s00216-014-8166-0) contains supplementary material, which is available to authorized users.

A. C. Chiaia-Hernandez • E. L. Schymanski • P. Kumar •

H. P. Singer $\cdot$ J. Hollender $(\triangle)$

Eawag, Swiss Federal Institute of Aquatic Science and Technology, Überlandstrasse 133, 8600 Dübendorf, Switzerland

e-mail: juliane.hollender@eawag.ch

A. C. Chiaia-Hernandez $\cdot$ J. Hollender

Institute of Biogeochemistry and Pollutant Dynamics, ETH Zurich,

Universitätstrasse 16, 8092 Zurich, Switzerland

P. Kumar

Faculty of Chemistry, University of Barcelona, Martí i Franquès, 1-11, 08028 Barcelona, Spain time/partitioning information and the number of references for a given substance. The combination of all available information resulted in the successful identification of three suspect (chlorophene, flufenamic acid, lufenuron) and two nontarget compounds (hexachlorophene, flucofuron), confirmed with reference standards, as well as the tentative identification of two chlorophene congeners (dichlorophene, bromochlorophene) that exhibited similar time trends through the sediment cores. This study demonstrates that complementary application of target, suspect, and nontarget screening can deliver valuable information despite the matrix complexity and provide records of historical contamination in two Swiss lakes with previously unreported compounds.

Keywords High-resolution mass spectrometry $\cdot$ Suspect screening · Nontarget screening $\cdot$ Sediment matrix · Organic contaminants $\cdot$ Mass accuracy $\cdot$ Chlorophenes

\section{Introduction}

Sediment cores are useful to reconstruct environmental changes over time, providing historic records of natural events, changes in ecosystems, climate, and anthropogenic impact. Thus, sediments can be suitable to reconstruct the environmental contamination within a catchment as they provide records of chemical deposition [1,2]. Additionally, it is now possible to use sediment cores or specimen banks to study the fate and exposure of many organic contaminants that could not be assessed in the past due to the lack of analytical technology available.

Different methods have been developed for the analysis of organic contaminants in sediments [2-5], and although these methods have been shown to be sensitive and selective, they aim to quantify and study only a small 
number of compounds. With the increasing number of synthetic compounds produced annually [6, 7], generic analytical techniques to measure a broader range of compounds in a single study are becoming essential to overcome the challenges in analyzing complex matrices that contain many target and nontarget compounds at different concentrations. The analysis of micropollutants in complex matrices, e.g., soils, sludge, and sediments, can be difficult since the presence of natural organic matter such as humic acids and biomolecules can interfere with the extraction and later on with the separation and detection [8]. High-resolution mass spectrometry (HRMS) using Orbitrap and time-of-flight (TOF) instruments is very suitable for these applications due to the high resolving power, mass accuracy, and sensitivity in full scan acquisition $[8,9]$. Generic analytical methods in combination with HRMS have been proven to reliably screen molecular ions and their MS/MS fragments against a complex sediment matrix background [1]. Since not all organic contaminants can be captured by target analysis alone, new approaches have been developed for the identification of compounds in surface water and wastewater without reference compounds using prior knowledge (i.e., suspect screening) or without any information (i.e., nontarget screening) [8, 10-15]. Ibáñez et al. [10] applied a suspect screening approach by comparing experimental data with a homemade spectral library containing 500 compounds. Moschet et al. [16] searched for all registered pesticides in Switzerland in surface waters using the exact mass followed by automatic filters for blank subtraction, peak area, peak shape, signal-to-noise ratio, and isotopic pattern with an overall success rate of $70 \%$. These approaches can help tentatively identify possible relevant contaminants without purchasing and measuring hundreds of reference standards in advance.

Structure elucidation can be achieved by applying a series of steps including the prediction of molecular formula from exact mass and isotope pattern match, followed by structure search in databases, matching retention time (RT) and MS/MS fragmentation [8]. Element information such as the presence of $\mathrm{Cl}, \mathrm{Br}$, or $\mathrm{S}$ has been successfully used to restrict possible molecular formulas due to their distinct isotope signal at $\mathrm{M}+2$ $[14,17]$ and characteristic mass defect compared with natural compounds $[8,18]$.

However, the identification of suspects and nontarget compounds is still an analytical challenge since software and methods to predict fragmentation patterns, ionization behavior, and retention time are still under development. Commercial software such as Mass Frontier [19] and Mass Fragmenter [20] are available to predict mass spectral fragments using different fragmentation rules, but Mass Frontier, for example, does not contain many rules for negative ionization. Wolf et al. [21] combined in silico bond disconnection fragmentation with compound database searching using KEGG [22], PubChem [23], and ChemSpider [24] in the openly accessible MetFrag. Other recent developments include CFM-ID [25]. Furthermore, the lack of comprehensive mass spectral libraries for high-accuracy MS/MS and the limited comparability between collision-induced dissociation (CID) and higher energy collision dissociation (HCD) spectra make the identification of unknown compounds more challenging. Recent contributions to the open spectral libraries like MassBank [26], mzCloud [27], and METLIN [28] are a step in the right direction. Here, it was hypothesized that in silico methods for structure elucidation and further exclusion steps based on physicochemical characteristics and patterns, MS/MS fragmentation, and ultimately confirmation with the purchase of reference standards could facilitate the identification of nontarget compounds as has been shown for GC-MS [29].

In a previous study, a multiresidue method for the target screening of more than 200 compounds with a broad range of physicochemical properties $\left(\log K_{\text {ow }} 0\right.$ 12) was developed [1] and applied to identify target compounds and screen for a few important suspects as the first step to identify potential organic contaminants that were not originally in the target list. The objectives of this study were to extend this previous work, namely (i) to explore suspect screening further using an automatic filtering for over 800 compounds, (ii) to identify nontarget compounds by performing in silico elucidation and candidate selection or exclusion for peaks with distinct negative mass defect, and thus, (iii) to clarify whether the suspect and nontarget screening methods could also be viable for complex sediment matrices and thus provide records of historical contamination in two Swiss lakes with previously unreported compounds.

\section{Experimental section}

Details on the sources, preparation, and storage of target reference standards and reagents are given in the Electronic supplementary material (ESM) and elsewhere [1]. The reference standards chlorophene, mefloquine, oxaprozin, fenazaquin, lumefantrine, mianserin, flunarizine, $p$ chlorocresol, lufenuron, flufenamic acid, and $p$ chlorophenol (purity $\geq 97 \%$ ) were purchased from SigmaAldrich (Buchs, Switzerland), simvastatin (purity $\geq 98 \%$ ) from TRC (Toronto, Canada), and flucofuron and hexachlorophene (purity $\geq 97 \%$ ) from Dr. Ehrenstorfer (Augsburg, Germany). 
Sample collection and preservation

Sediment cores were taken from Lake Greifensee $\left(47^{\circ} 20^{\prime} 58^{\prime \prime}\right.$ $\left.\mathrm{N}, 8^{\circ} 40^{\prime} 49^{\prime \prime} \mathrm{E}\right)$ and Lake Lugano (45 $57^{\prime} 31.5^{\prime \prime} \mathrm{N}, 8^{\circ} 53^{\prime}$ $\left.38.3^{\prime \prime} \mathrm{E}\right)$ located in the north and south of Switzerland, during the summer of 2010 and 2012. Sediment core samples were obtained using a free fall gravity corer and stored vertically in the dark at $4{ }^{\circ} \mathrm{C}$ until analysis. Dating of the sediment cores ( 1950-2012) was performed by counting annual laminations and by using ${ }^{137} \mathrm{Cs}$ signals from Chernobyl 1986 and atomic bomb tests from 1963, as described elsewhere [1].

Liquid chromatography tandem high-resolution mass spectrometric detection

Sediment samples were freeze-dried, extracted by pressurized liquid extraction, and cleaned up by liquid-liquid partitioning using a combination of acetonitrile, $\mathrm{MgSO}_{4}$, and $\mathrm{NH}_{4} \mathrm{Cl}$ as reported elsewhere [1]. Detection of analytes was performed with a linear ion trap Orbitrap (LTQ-Orbitrap-XL) and a quadrupole Orbitrap (Q-Exactive) mass spectrometer (Thermo Fisher Scientific Corp., USA). Electrospray ionization (ESI) was performed using positive and negative ionization modes in multiple independent injections to acquire meaningful MS/MS spectra. Full scan accurate mass spectra were acquired from 115 to $1,000 \mathrm{Da}$ with a resolving power of 60,000 referenced to $\mathrm{m} / \mathrm{z} 400$ and a mass accuracy of $\pm 5 \mathrm{ppm}$. Data-dependent high-resolution product ion spectra (DD-HR-MS/MS) were recorded at a resolving power of 7,500 for LTQ-Orbitrap-XL. Q-Exactive spectra were acquired at a resolving power of 100,000 referenced to $\mathrm{m} / \mathrm{z} 400$ and a mass accuracy of $\pm 5 \mathrm{ppm}$ and a DD-HR-MS/MS of 17,500.

Product ions were generated using HCD at collision energies calculated for each analyte based on its mass. In addition, CID was performed with a normalized collision energy of $35 \%$, measured at low resolution in the linear ion trap. Calibration standards, quality controls, and quantification criteria used in the analysis are reported in the ESM.

Limits of quantification (LOQ) were defined as the lowest point of the calibration curve with a signal-to-noise $(\mathrm{S} / \mathrm{N})$ ratio $\geq 10$ and with sufficient ions for MS/MS fragmentation. The LOQ were then divided by a calculated matrix factor for each compound and subsequently multiplied by the dilution of the matrix sample. The matrix factor and calculated matrix effect (\%) for each analyte are reported elsewhere [1].

Suspect screening of contaminants and transformation products

A suspect list of 838 contaminants was compiled from a theoretical assessment based on consumption data, including all registered organic synthetic insecticides, fungicides, biocides, and acaricides, including all major metabolites of the most commonly used insecticides and fungicides in Switzerland [16] as well as important pharmaceuticals used in Switzerland, Germany, France, and the USA (Singer et al., in preparation) which were not yet included in the target list. As only more hydrophobic compounds were expected to partition into sediments, the list of suspect compounds was narrowed to candidates with predicted $\log K_{\mathrm{ow}}$ values higher than 1 using VCCLAB software [30]. Further, suspect compounds were eliminated if they were not likely to be ionized by ESI using rules (presence or absence of functional groups) as in Moschet et al. [16]. The complete list of suspect compounds that were estimated to fall in the domain of the analytical method is provided in ESM Table S1.

Suspect screening was performed in sediment core layers between 1950 and 2012 from Lake Greifensee and Lake Lugano with the aid of ExactFinder 2.0 (Thermo Fisher Scientific Corp., USA). The exact mass of the expected ion was extracted from the HR full scan chromatogram using a mass window of $\pm 5 \mathrm{ppm}$. As ESI generally produces molecular ions $[\mathrm{M}+\mathrm{H}]^{+}$or $[\mathrm{M}-\mathrm{H}]^{-}[31]$, suspect screening was performed using these masses. Other adducts (e.g., $\mathrm{NH}_{4}{ }^{+}, \mathrm{Na}^{+}, \mathrm{HCO}_{2}{ }^{-}$, and $\mathrm{H}_{3} \mathrm{C}_{2} \mathrm{O}_{2}{ }^{-}$) were not included for simplicity. Due to the difficulty of predicting ionization behavior, all suspected targets were screened in positive and negative ionization modes to avoid losing compounds due to an incorrect prediction in ionization at the beginning of the workflow. Blank subtraction was performed using a sediment layer from $\sim 100$ years ago for each core, as contamination was not considered likely to be present in these layers. Additional automatic filtering criteria included peak area cutoff $\left(\geq 10^{4}\right.$ in negative and $\geq 10^{5}$ in positive), $\mathrm{S} / \mathrm{N}(\geq 50)$, peak score $(\geq 0.45)$, and isotopic pattern $(\geq 50)$.

The output list of suspect candidates was then evaluated manually for correct isotopic pattern, $\mathrm{S} / \mathrm{N}$, peak shape, and ionization in the positive and negative modes (based on different functional groups and literature). A RT match factor with a window of \pm 4 min was also included in the analysis. Predicted RTs were obtained using a linear correlation between the measured retention times and predicted $\log K_{\mathrm{ow}}$ values $(\mathrm{RT}=1.65 \times \log$ $\left.K_{\mathrm{ow}}+4.36\right)$ obtained from the 164 reference standards used in the target analysis [1].

To obtain more information on suspect candidates, samples were re-injected and measured at different collision energies (HCD) of 15, 35, and $55 \%$ for targeted MS/MS fragmentation. MS/MS spectra were compared with predicted MS/MS fragmentation using Mass Frontier 6.0 [19] and MetFrag [21] using ChemSpider and PubChem to perform compound database searches and 
in silico fragmentation for the molecular formula of the suspect candidate. Unequivocal confirmation was only possible if a reference standard was available; where standards were available, the MS/MS spectra are saved in MassBank (www.massbank.eu/MassBank/).

\section{Nontarget screening of contaminants}

Nontarget screening was performed in the most recent layers (the last 10 years) and the most contaminated layers of each core. The highest period of contamination around $\sim 1980$ s for Lake Greifensee [1] and between $\sim 1970$ and $\sim 1980$ s for Lake Lugano is clearly distinguishable from the other layers. A nontarget mass list for each sediment sample was obtained using enviMass 1.2 [32], with peak picking performed using Formulator (Thermo Fisher Scientific Corp., USA). Nontarget candidates were selected using an automated exact mass filter with a window of \pm 5 ppm, followed by a background subtraction and recalibration of masses using internal standards. A sediment layer $(\sim 100$ years ago $)$ within each core was set as a background sample to eliminate matrix peaks. Additionally, background subtraction was performed using sediment core layers adjacent to the layers of interest with the aim to match the sediment matrix as close as possible and to identify the most relevant (intense) unknown masses. For example, the layer corresponding to the 1990s (layer 2) was used as a background and subtracted from the first layer of each sediment core (the last 10 years).

Furthermore, the nontarget list was reduced by selecting the top 10 most intense masses from each sediment layer and the top 100 masses containing $\mathrm{Cl}$, $\mathrm{Br}$, and $\mathrm{S}$ based on isotopic information obtained from enviMass. Masses higher than $\mathrm{m} / \mathrm{z} 600$ were excluded since most contaminants have lower molecular masses (e.g., only $3 \%$ of the target and suspect list was above this mass) and because many of the high masses are considered likely to belong to the sample matrix. The nontarget list was reduced further by keeping candidates containing mass defect between -200 and $0 \mathrm{mDa}$ to focus on molecular formulas outside the heavy matrix domain. As for suspect confirmation, the final nontarget candidates were re-injected and measured at different collision energies (HCD) of 15, 35, and $55 \%$ for targeted MS/MS fragmentation; the reference spectra are also available at www.massbank.eu/MassBank/.

Structure elucidation using the full scan and MS/MS data was performed using MOLGEN-MS/MS [33] and MetFrag using ChemSpider and PubChem. MOLGENMS/MS was used with a mass accuracy of 5 and $10 \mathrm{ppm}$ in the MS and MS/MS spectra, respectively, checking for the existence of at least one structure for the given formula (exist filter) and considering odd and even ion species for fragments. Additional parameters were adjusted to the individual cases and included element restrictions (e.g., C, H, N, O, P, S, including $\mathrm{Cl}$ and $\mathrm{Br}$ if halogen patterns were detected) and the ion species (e.g., $[\mathrm{M}+\mathrm{H}]^{+}$or $[\mathrm{M}-\mathrm{H}]^{-}$). MetFrag was employed using the neutral exact mass (with mass error $5 \mathrm{ppm}$ ) or molecular formula for candidate retrieval with the appropriate ionization mode and charge, while in silico fragmentation was performed using a combined relative mass error of $5 \mathrm{ppm}$ and absolute error of $0.001 \mathrm{Da}$.

\section{Results and discussion}

Target screening of organic contaminants

Comprehensive target analysis is essential before suspect and nontarget screening in order to clearly concentrate on previously unknown compounds as shown by Krauss et al. [8]. In Lake Lugano located in the south of Switzerland, 16 of 200 target compounds were detected with the most prominent substance classes corresponding to musk fragrances, personal care products (PCP), pesticides, and biocides, similar to the previously described results for Lake Greifensee located in the north of Switzerland [1]. In both lakes, the musk fragrance tonalide, the biocides triclosan and triclocarban, and the quaternary ammonium surfactants, congeners benzyldimethyltetradecylammonium (BAC 14) and didodecyldimethylammonium (DADMAC C12:C12), show similar patterns with increasing concentrations in the late 1970s, followed by a decline in concentrations starting in the 1990s. The biocides irgarol, terbutryn, and prometryn and a transformation product of the musk fragrance galaxolide (galaxolidone) were detected in the nanogram per gram dry weight to the picogram per gram dry weight range with concentrations increasing through the early 1990s and with the highest concentrations in the most recent layers. The biocide propiconazole as well as the pesticides fludioxonil, DEET, and the co-eluting compounds acetochlor plus alachlor (isobaric; total concentrations reported) was detected in layers of the past $\sim 10$ years. The quantified concentrations for all positive findings in Lake Lugano are reported in Table 1. While little information was available about the historical contamination of Lake Lugano previously, it was known that the lake was highly contaminated in the 1960s and 1970s [34], similar to Lake Greifensee. The concentration range $\left(\mathrm{pg} / \mathrm{g}_{\mathrm{dw}}\right.$ to $\left.\mathrm{ng} / \mathrm{g}_{\mathrm{dw}}\right)$ and contamination pattern in both lakes are quite consistent. 


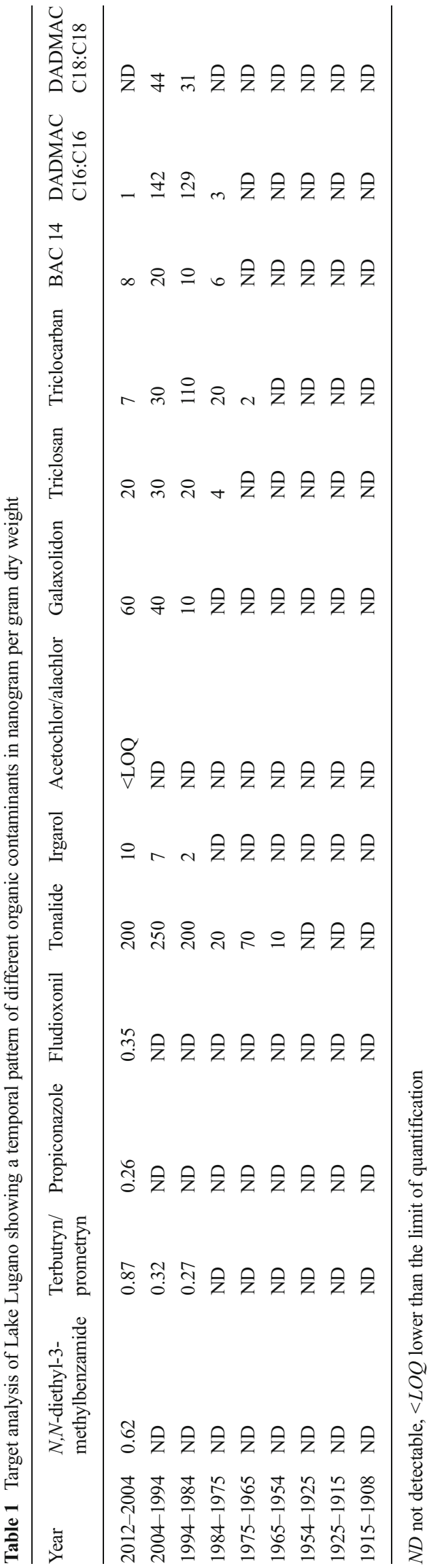

Suspect screening

The detection of organic contaminants was expanded beyond the target list to include 838 suspect candidates. To compare the candidates with that of the complex sediment matrix, a mass list for each lake sediment was compiled using sediment core layers corresponding to the $\sim 1950$ s for both lakes. The mass defect (mDa) versus nominal masses $(\mathrm{m} / \mathrm{z})$ was plotted for the retention time window $10-15 \mathrm{~min}$ as shown in Fig. 1. For most of the matrix ions (black dots), a slightly positive mass defect is observed, increasing with the nominal mass and consistent with natural compounds containing $\mathrm{C}, \mathrm{H}, \mathrm{N}$, and $\mathrm{O}$ atoms such as fatty acids, peptides, polyphenols, carbohydrates, and humic acids. The suspect compounds (red dots) generally fall in the same domain as the sediment matrix, which increases the presence of interferences during identification efforts. Further, the matrix was more predominant in negative compared with positive ionization, which is reasonable as many natural compounds are acids.

The automated data processing with ExactFinder (peak shape and intensity, blank subtraction, retention time) allowed the elimination of an average of $80 \%$ possible suspect candidates, while visual inspection of chromatograms reduced the suspect candidate list further by 3 to $20 \%$. Suspects for which the intensity ratio of the isotope pattern differed by more than $50 \%$ from the theoretical pattern were eliminated. In the absence of a distinct isotopic signal, an intensity cutoff of $10^{6}$ was performed to focus on the most prominent suspect compounds, analogous to Moschet et al. [16]. As a last step, candidates outside a predicted RT with a large error window $( \pm 4 \mathrm{~min})$ were eliminated despite the limitations inherent in this method since large errors in the predicted $\log K_{\text {ow }}$ are involved, especially for ionic compounds. However, in-house experience with target analysis using different $\log K_{\text {ow }}$ predictions demonstrated that the target compounds fell consistently within the RT window of $\pm 4 \mathrm{~min}$, which is also within the boundaries of the $95 \%$ prediction interval of the linear correlation used [1]. This filter step was not applied to suspect candidates that were expected to be protonated or deprotonated under the analytical conditions used.

In total, 27 of 839 exact mass matches were selected for further analysis. The MS/MS fragmentation of the selected candidates was acquired, and a score based on the number and intensity of matching-predicted fragments was obtained using MetFrag. Candidates with a score of 0 (maximum value=1) and with 0 matching fragments were not considered further. For the remaining compounds, reference standards were purchased 

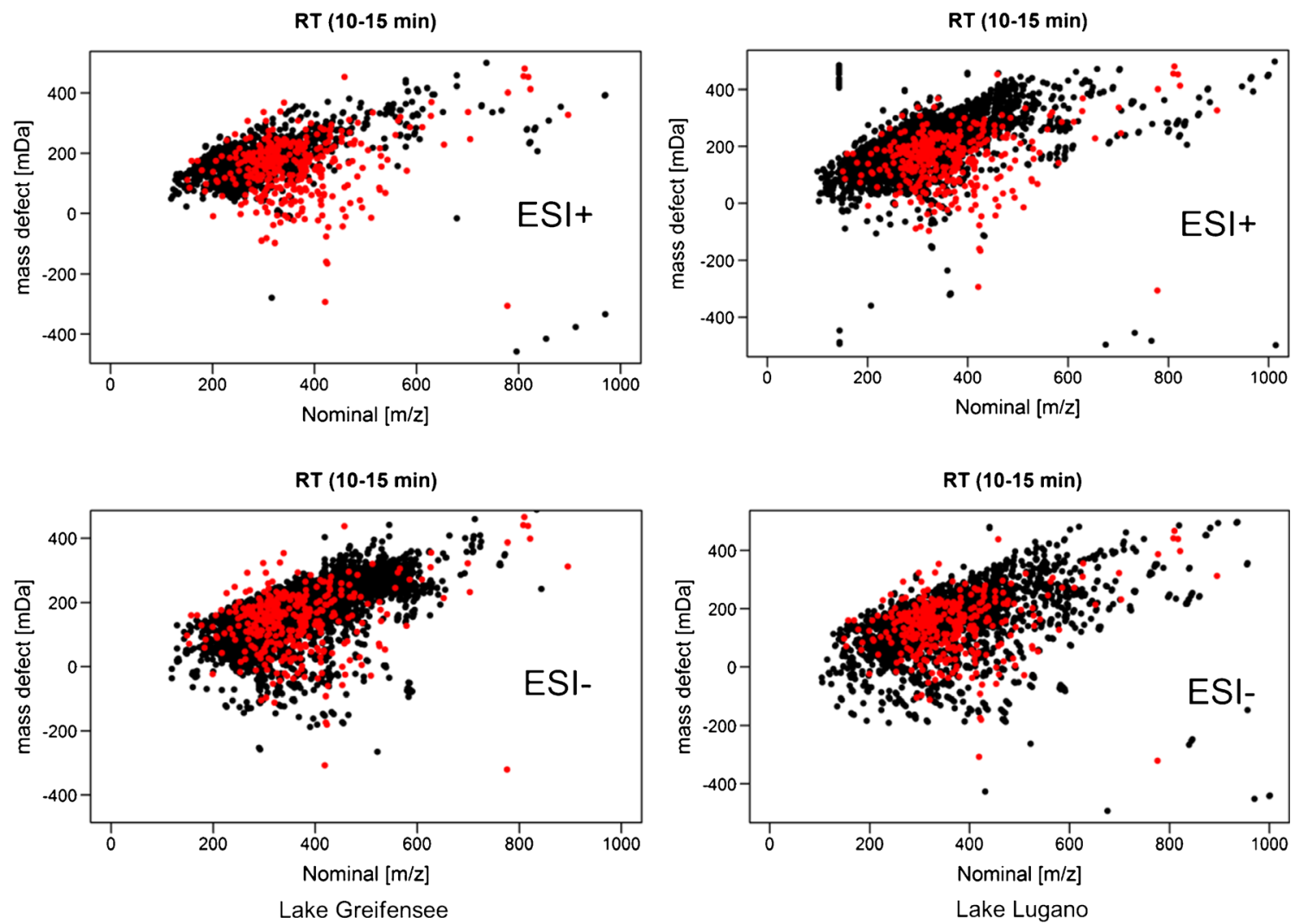

Fig. 1 Mass defects $(\mathrm{mDa})$ of Lake Greifensee and Lake Lugano sediment matrix (black dots) in positive and negative ionization modes within the retention time window of $10-15 \mathrm{~min}$ are plotted in the $y$-axis against

where easily available (8 of 13 compounds), as illustrated in Table 2.

The biocide chlorophene was detected in both lakes and tentatively identified using MetFrag with four matching fragments in the MS/MS spectrum with a score of 0.987 and finally confirmed with a reference standard (Table 2 and ESM Fig. S1). The pattern of chlorophene in both lakes shows the highest input in the $\sim 1980$ s and $\sim 1990$ s for Lake Greifensee and Lake Lugano, respectively, as illustrated in Fig. 2. Additionally, the pharmaceutical flufenamic acid and the pesticide lufenuron were detected and confirmed by reference standards in both lakes.

While the evaluation of experimental MS/MS spectra with predicted MS/MS fragmentation using Mass Frontier and MetFrag was certainly helpful for the exclusion of candidates in the sediment matrix, confirmation of the tentative candidates via reference standards was shown to be important. The pesticide fenazaquin had a MetFrag score of 1 (with seven matching fragments), but the MS/MS of the reference standard did not match the measured spectrum, with one main fragment absent. Further, as three of the five unconfirmed suspect compounds have a mass defect between 180 and $265 \mathrm{mDa}$ nominal $(\mathrm{m} / \mathrm{z})$ masses at the $x$-axis. Masses of suspect compounds are plotted as red dots

and thus fall into the matrix domain (Fig. 1), matrix interferences may have resulted in additional peaks in the MS/MS.

\section{Nontarget screening}

enviMass proved a useful first step for the identification of nontarget candidates from samples with an average of $17,000 \pm 4,000$ picked peaks, as background subtraction and noise removal eliminated up to $60 \%$ of these picked peaks, shown in Fig. 3. Overall, maximal $2 \%$ of the picked masses were known (target compounds and internal standards), while up to $52 \%$ of the masses remained unknown. For all sediments, the nontarget candidates contained between 9 and $18 \%$ peaks with an isotopic pattern, 1-5\% with adducts, and around $80 \%$ monoisotopic peaks (Fig. 3). As only a single mass is available for monoisotopic peaks and these are generally of low intensity, structure elucidation was not attempted for monoisotopic peaks. While the results were generally similar for negative and positive ionization for both lakes, a higher percentage of unknowns (52\%) were found for the negative mode in Lake Greifensee. This could be explained by the stronger 


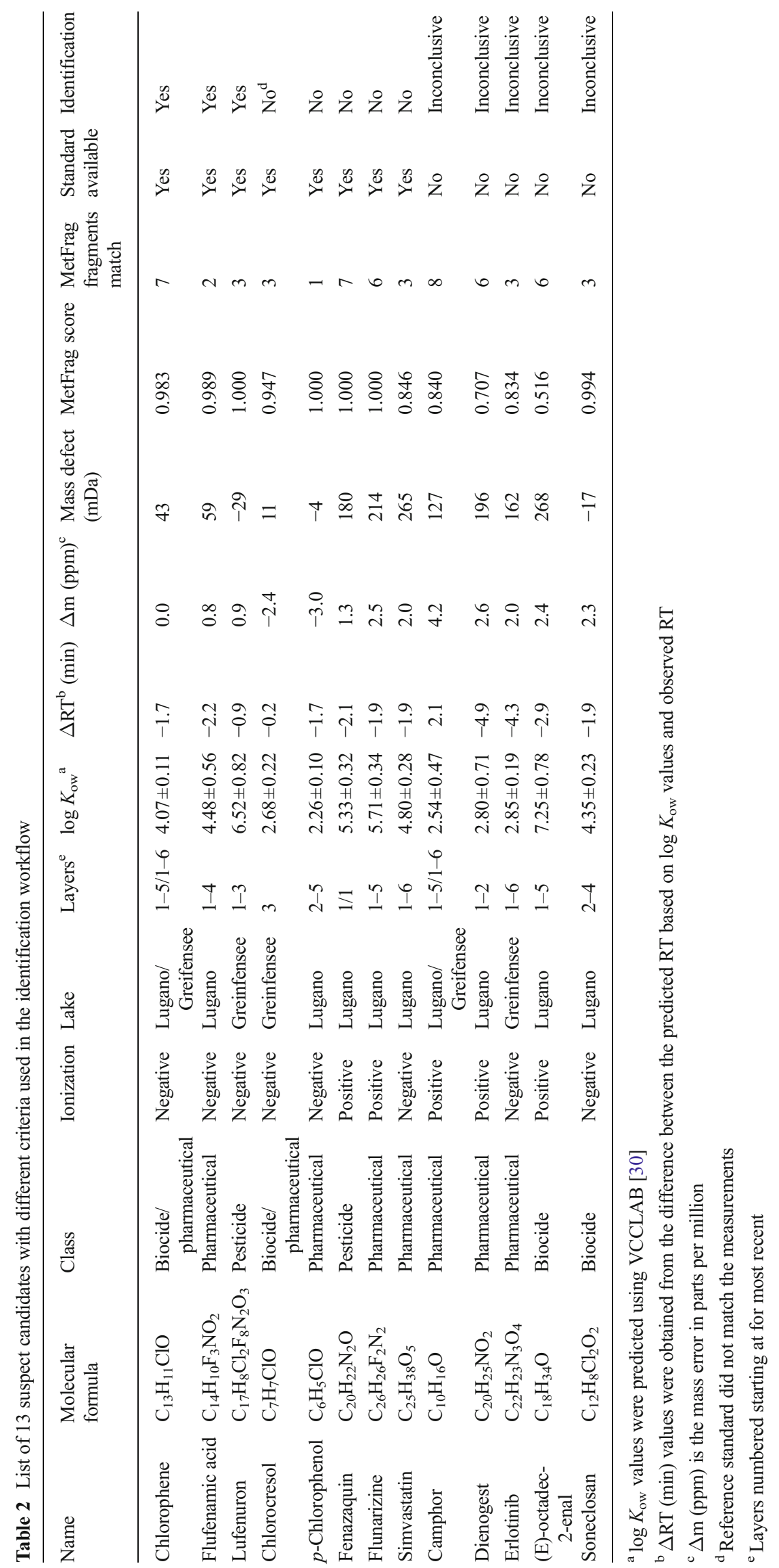




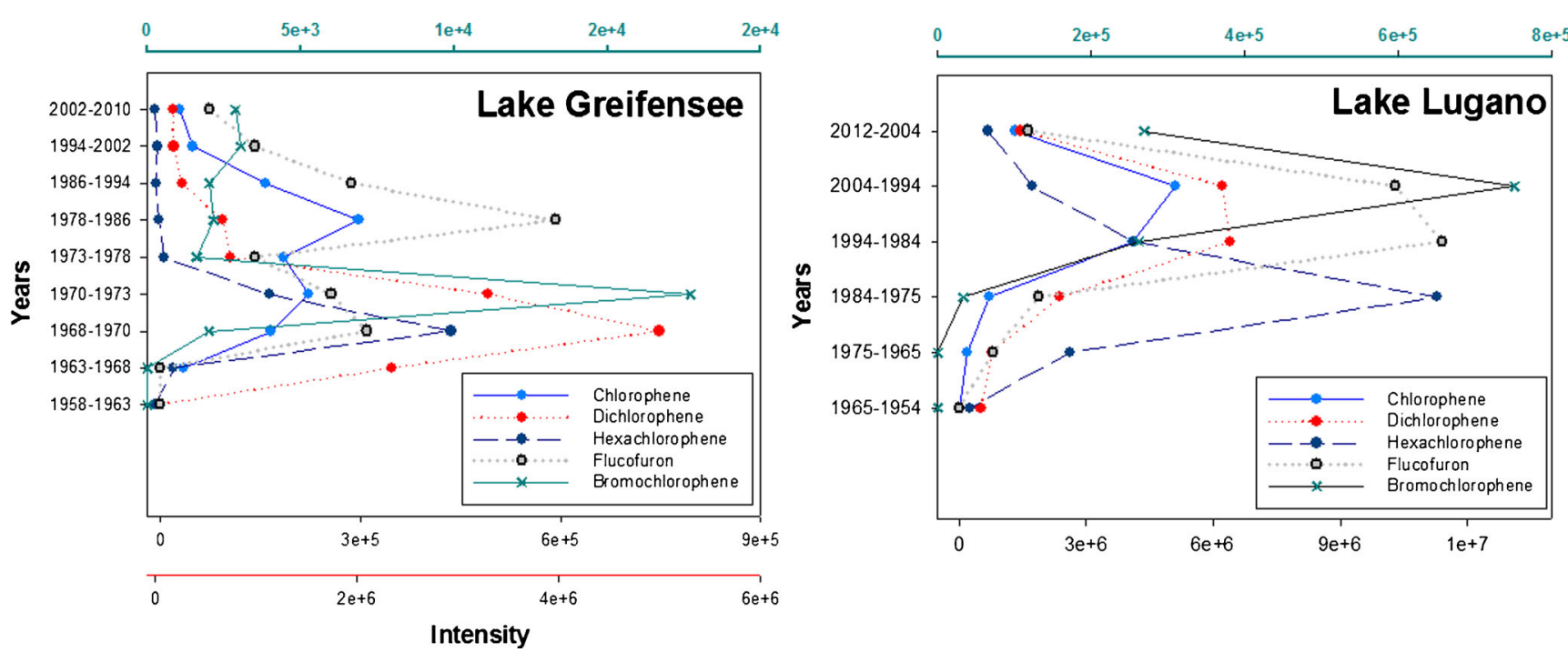

Fig. 2 Temporal resolution of suspect and nontarget compounds in sediments from Lake Greifensee and Lake Lugano

matrix observed in the negative measurements in Lake Greifensee (Fig. 1), compared with the positive measurement. As an exact match matrix blank subtraction was not possible for this environmental sample, the high number of unknown peaks could correspond to natural organic matter and macromolecule masses that were not present in the 100 year old sediment layer. Additionally, more nontarget candidates containing isotope signals associated with $\mathrm{Cl}, \mathrm{Br}$, or $\mathrm{S}$ were observed in negative mode than in positive ionization. For example, in Lake Greifensee, only 30 exact masses containing $\mathrm{Cl}, \mathrm{Br}$, or $\mathrm{S}$ were found in positive ionization, while 740 were in negative mode.

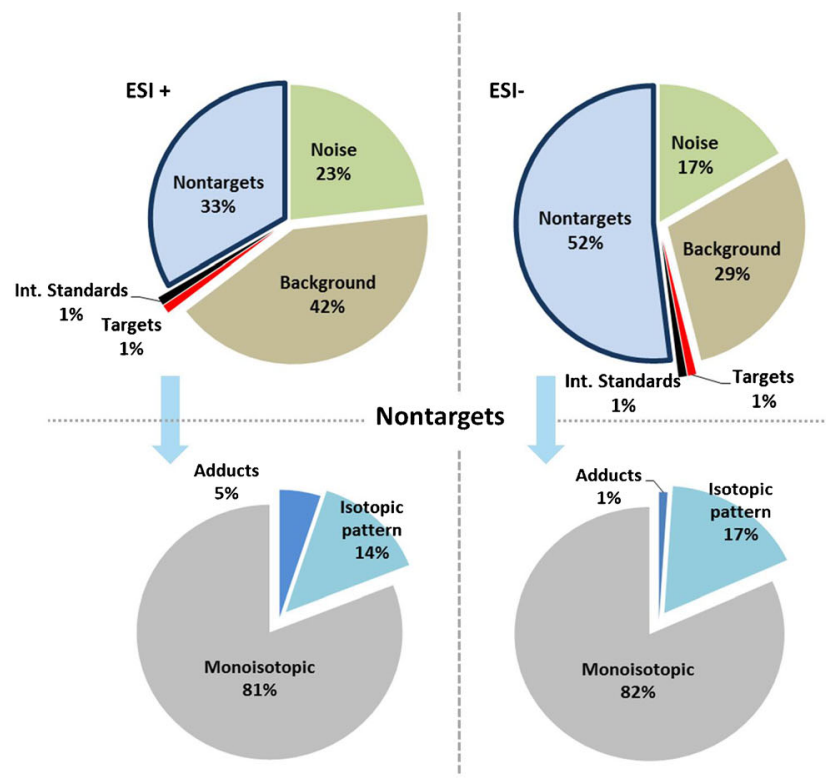

Lake Greifensee

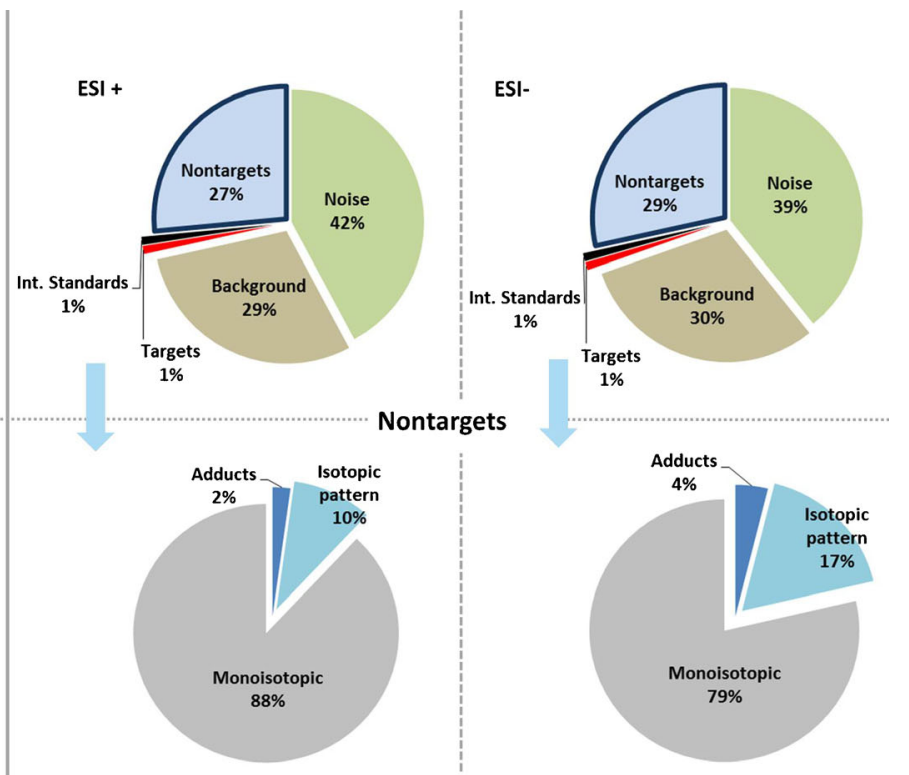

Lake Lugano
Fig. 3 Pie chart analysis of the most contaminated sediment layers ( 1970s) from Lake Greifensee and Lake Lugano based on enviMass outputs. Black, red, brown, green, and blue colors (shown at the top) represent the percentage of internal standards, targets, background, noise, and nontargets, respectively. Further nontarget analysis (shown at the bottom) represents the percentage of monoisotopic, isotopic, and adduct peaks displayed in gray, light blue, and dark blue, respectively 
Nontarget candidates with distinct isotope signals and exhibiting mass defects outside the matrix distribution (Fig. 1) were prioritized for further elucidation. The elucidation efforts are demonstrated with two nontarget masses $(\mathrm{m} / \mathrm{z}$ 402.8427 and 414.9846).

\section{Nontarget compound 1}

For the first nontarget compound, two molecular formulas were generated with MOLGEN-MS/MS restricted using all information available to give a top match with $\mathrm{C}_{13} \mathrm{H}_{6} \mathrm{Cl}_{6} \mathrm{O}_{2}$ (score 0.96). For this formula, 11 structures were retrieved by MetFrag, which explained two to three fragments with the proposed structures, with two fragments common to all 11 candidates. MOLGEN-MS/ MS identified subformulas for four of the fragments (Fig. 4). As all candidates were within the $\log K_{\text {ow }} /$ RT range, the nontarget candidates were arranged according to the number of references in ChemSpider and PubMed, shown previously to be very useful for the identification of "known unknowns" [35], which would be expected in historical sediments. Hexachlorophene topped this list with 909 references in ChemSpider and 657 in PubMed and was finally confirmed by the purchase of a reference standard as shown in Fig. 4. Hexachlorophene has been detected in estuary sediments in the USA [36], and although it is still in use as a surgical hand scrub and a bacteriostatic skin cleanser, there are only a few limited studies about its fate [37].

\section{Nontarget compound 2}

For nontarget compound 2, elucidation efforts using the measured $\mathrm{m} / \mathrm{z} 450.9619$ did not yield satisfactory molecular
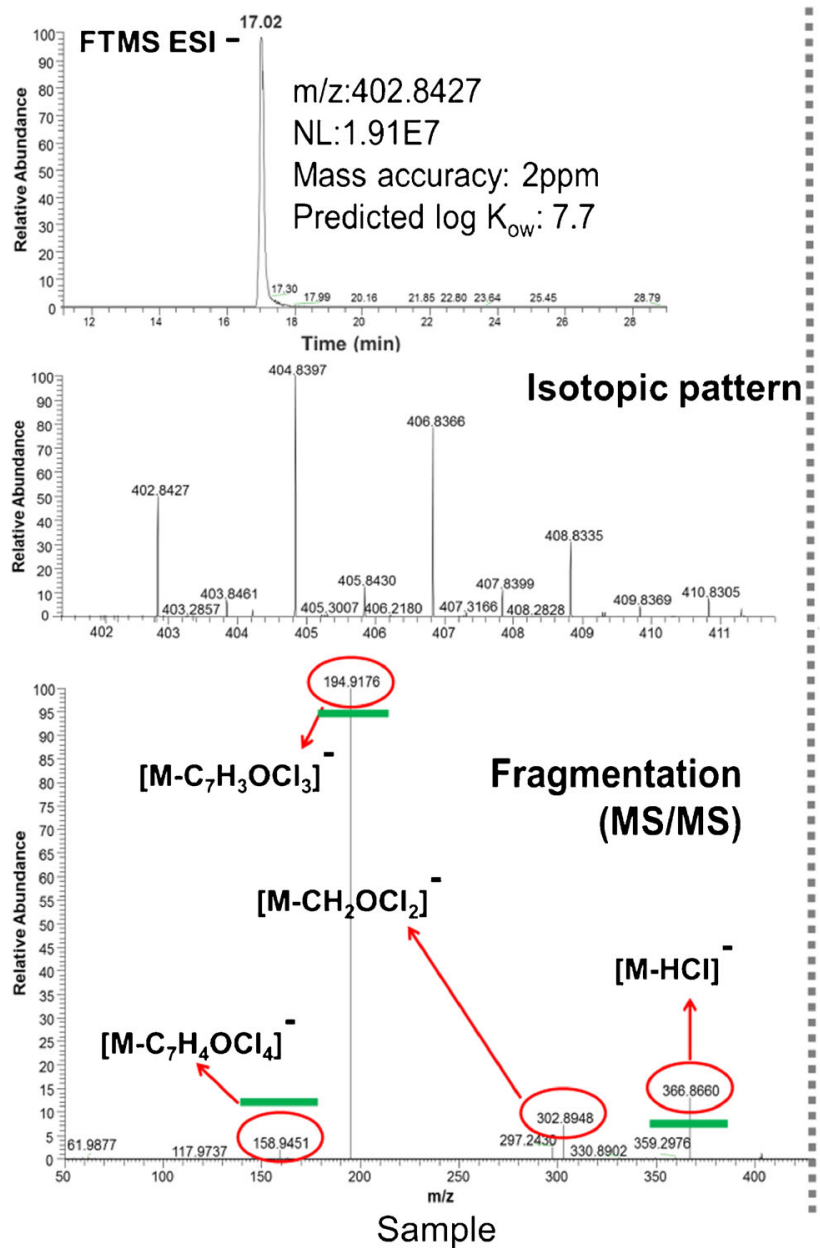

Fig. 4 Chromatogram, isotopic pattern, and MS/MS fragmentation used for the identification of the disinfectant hexachlorophene. The four fragments circled in red were identified successfully by MOLGEN-MS/MS.

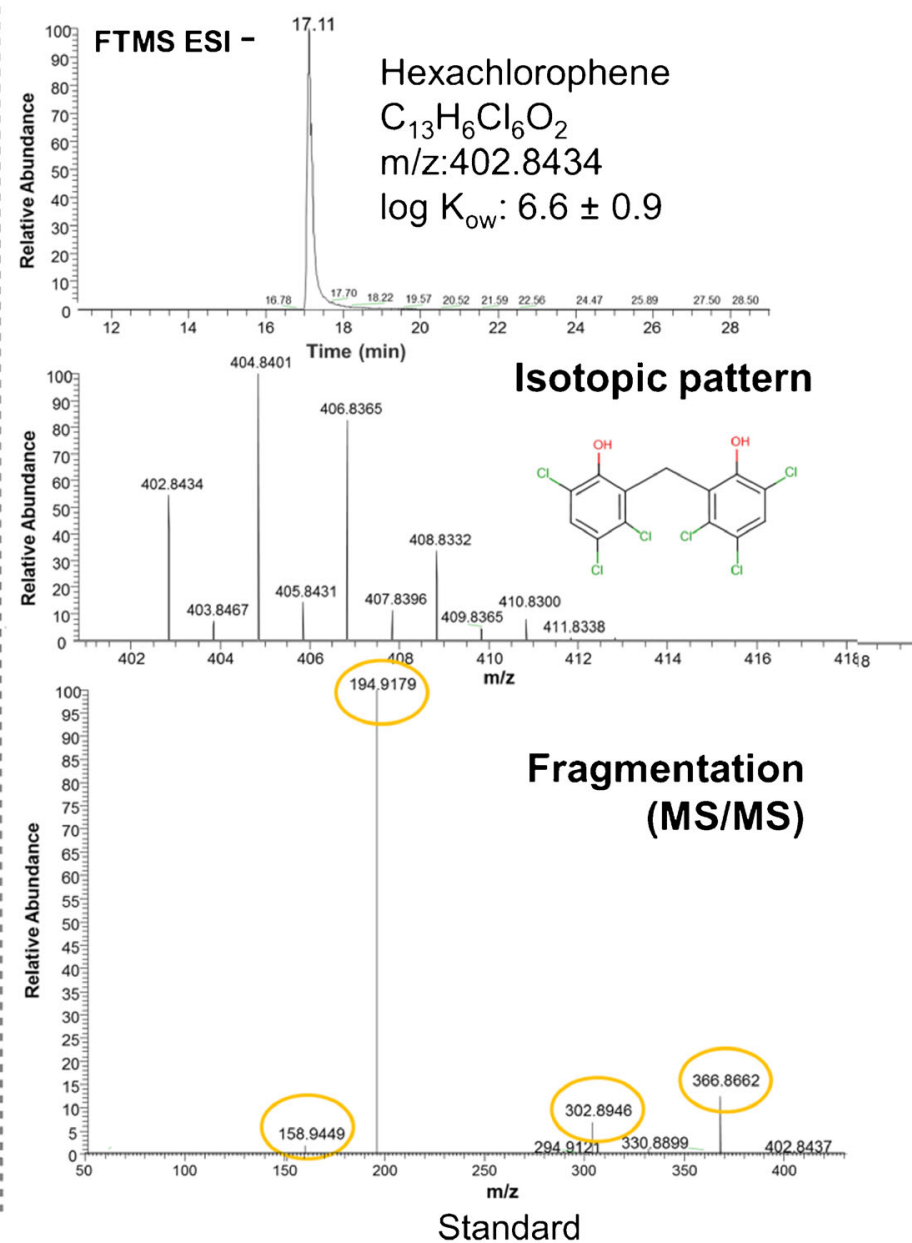

The three fragments marked in green were identified by MetFrag. The standard fragments are circled in yellow 
formula or structural candidates. Exploring different adduct options with MetFrag revealed many promising candidates when considering the chlorine adduct $[\mathrm{M}+\mathrm{Cl}]^{-}$. Consequently, the corresponding $[\mathrm{M}-\mathrm{H}]^{-}$exact mass, $m / z$ 414.9846, was searched in the chromatogram, revealing a more intense peak $\left(10^{6}\right.$ vs $\left.10^{5}\right)$ with the same retention time and peak shape as the chloride adduct. Furthermore, the fragmentation patterns were similar, with the $[\mathrm{M}+\mathrm{Cl}]^{-} \mathrm{MS} / \mathrm{MS}$ showing one additional fragment ( $\mathrm{Cl}$ loss) to yield the original compound ([M $-\mathrm{H}]^{-}$), confirming the assumption of the adduct formation. Although the formation of ion adducts in negative ESI has not been widely reported in literature compared with positive ESI, chloride adducts have been used to enhance ion abundances after addition or contamination with solvents or salts, and chloride adducts are reported to be one of the molecular ion adducts often observed in ESI-negative mass spectra. This is in agreement with our observations and the potential presence of residual $\mathrm{NH}_{4} \mathrm{Cl}$ from the QuEChERS sample cleanup [38-41].

The measured $\mathrm{m} / \mathrm{z}$, MS, and MS/MS spectra corresponding to $[\mathrm{M}-\mathrm{H}]^{-}$, as well as elemental restrictions $(\mathrm{C}, \mathrm{H}, \mathrm{O}, \mathrm{N}, \mathrm{Cl}, \mathrm{F})$, were used in MOLGEN-MS/MS to yield a top-scoring formula of $\mathrm{C}_{15} \mathrm{H}_{8} \mathrm{Cl}_{2} \mathrm{~F}_{6} \mathrm{~N}_{2} \mathrm{O}$ (score 0.96 ) as one of 140 possible formulas due to the presence of F. This formula was used in MetFrag to retrieve five structures, where two had a score of 1.0, shown in Fig. 5. The top 2 candidates varied only in the position of a chloride within one of the benzene rings, with the number of references indicating that the leftmost candidate in Fig. 5, flucofuron, was more likely (13 references in ChemSpider). Confirmation was achieved using a reference standard as shown in Fig. 6. While flucofuron is not currently available commercially in any mothproofing agents [42], it can still be detected in sediments due to historical use. The temporal resolution of flucofuron and hexachlorophene in the sediment cores is illustrated in Fig. 2.

The negative mass defects of hexachlorophene and flucofuron, respectively, mean that these compounds fall outside of the sediment matrix. As shown in Fig. 1, this reduced the number of interfering peaks in the MS and MS/MS (within the isolation window of $1.5 \mathrm{Da}$ ), dramatically improving both the MS1 isotope pattern match and fragmentation prediction and assignment.
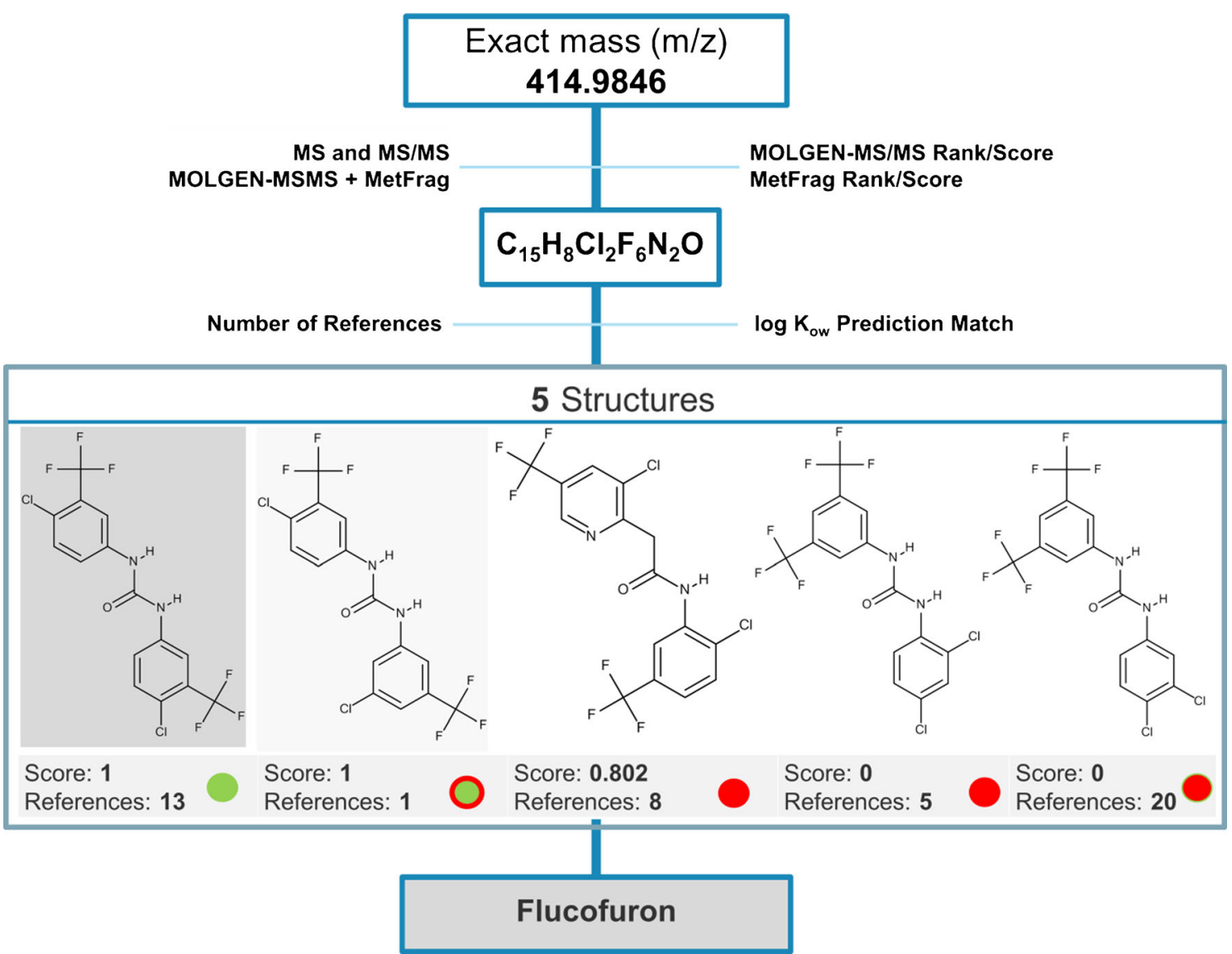

Fig. 5 Flowchart for the identification of the mothproofing agent flucofuron. Criteria used for the identification is illustrated at each step, score values are from MetFrag, Reference numbers from ChemSpider 
FTMS ESI-
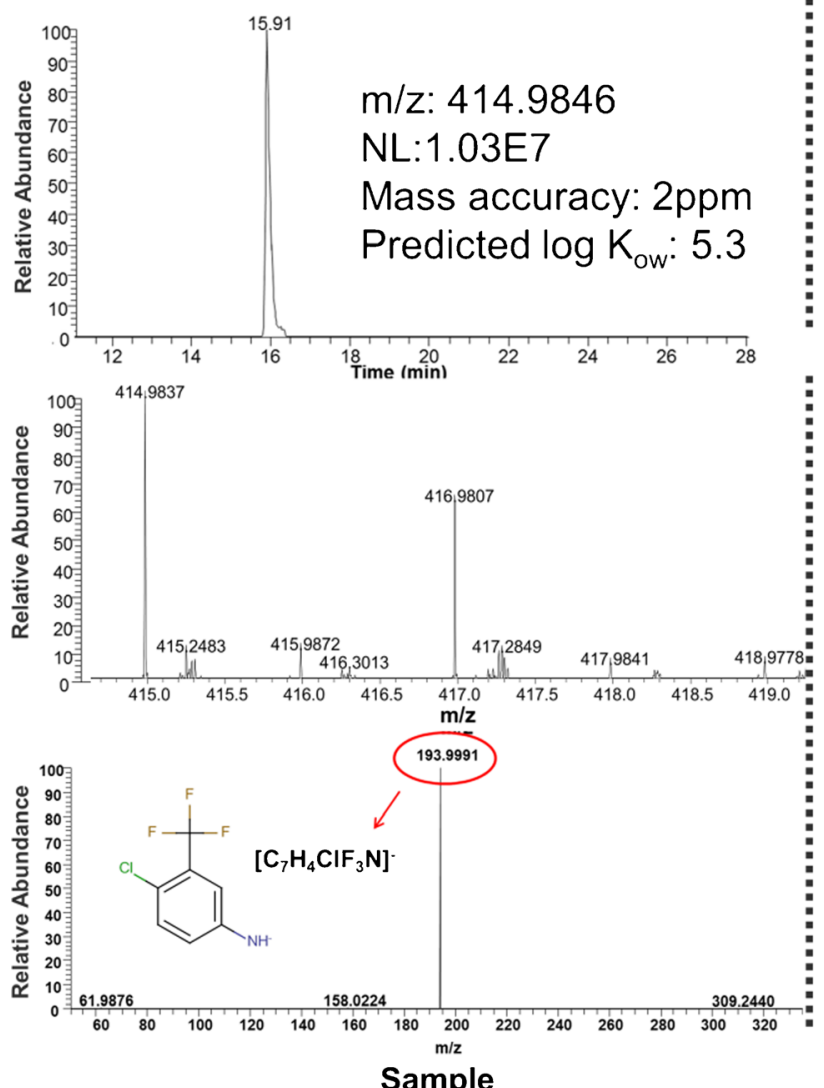

FTMS ESI-
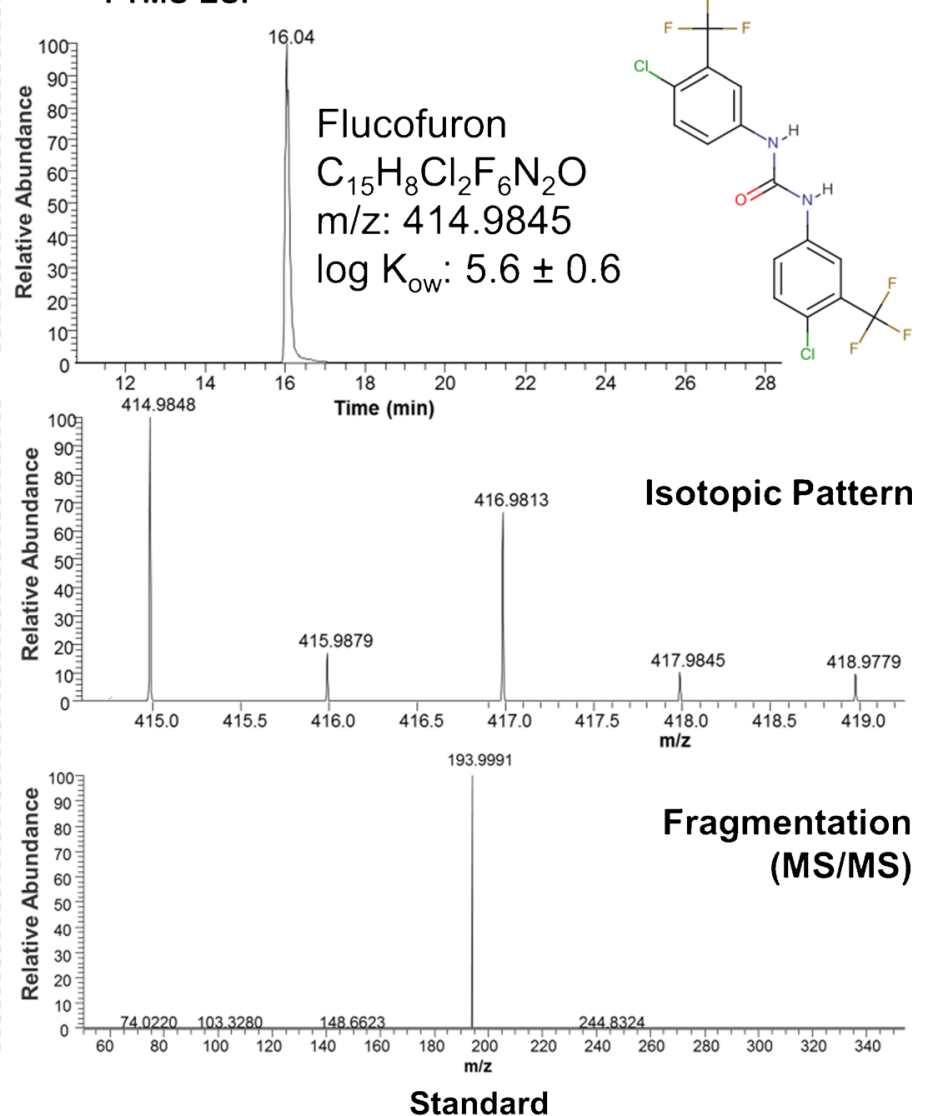

Fig. 6 Chromatogram, isotopic pattern, and MS/MS fragmentation used for the identification of the mothproofing agent flucofuron

\section{Detection of congeners and related compounds}

The suspect and nontarget screening analysis revealed two disinfectants from one substance class: chlorophene and hexachlorophene. Based on these results, further chlorophene and bromophene congeners were screened in Lake Lugano and Lake Greifensee, yielding tentative identifications of the biocides dichlorophene and bromochlorophene. Bromochlorophene is very similar to tetrachlorophene, with one $\mathrm{Cl}$ per aromatic ring replaced with $\mathrm{Br}$ in bromochlorophene. The presence of a characteristic fragment (see ESM Fig. S2) gives additional evidence to support the presence of an isomer of bromochlorophene, but reference standards are not available for all isomers and the fragmentation pattern did not match completely with the one standard purchased. Both compounds exhibited a similar temporal pattern to hexachlorophene and chlorophene in Lake Greifensee and Lake Lugano, respectively (Fig. 2). The results here show that screening for related compounds, as for chlorophene and its halogenated congeners, can also be a successful suspect screening strategy.

\section{Conclusions}

The analysis of micropollutants in sediments is generally more challenging than water samples due to the higher occurrence of natural organic matter and biomolecules, which can interfere with the analysis of organic contaminants. The strategy to search for compounds with a clear isotopic pattern $(\mathrm{Cl}, \mathrm{Br})$ and mass defects outside the prominent matrix mass range facilitated the suspect and nontarget screening and was very successful. The combination of MOLGEN-MS/MS and MetFrag helped direct identification efforts toward "known unknown" compounds and thus provide records of the historical contamination of two Swiss lakes. In total, three suspect candidates, two nontarget compounds, and one congener of a suspect were confirmed with reference standards, while a further congener was tentatively identified. The bias here toward halogenated compounds is reasonable as many anthropogenic and potentially toxic compounds in the environment contain halogens and thus fall outside the sample matrix, but future research should address also the challenges in identifying toxicants without halogens in complex matrices. 
Acknowledgments The authors are grateful for the assistance provided by Birgit Beck (sediment analysis), Martin Loos (enviMass), Lee Ferguson (input in suspect and nontarget screening), Martin Frey (R), Markus Moest, Piet Spaak, and Flavio Anselmetti (sediment core collection). Funding from the Swiss National Science Foundation (SNF CR32I3 125211), the European Union (Marie Curie Inter-European Postdoctoral Fellowship Grant No. 299734), and the Swiss Federal Office for the Environment is gratefully acknowledged.

\section{References}

1. Chiaia-Hernandez AC, Krauss M, Hollender J (2012) Screening of lake sediments for emerging contaminants by liquid chromatography atmospheric pressure photoionization and electrospray ionization coupled to high resolution mass spectrometry. Environ Sci Technol 47:976-986

2. Peck AM, Linebaugh EK, Hornbuckle KC (2006) Synthetic musk fragrances in Lake Erie and Lake Ontario sediment cores. Environ Sci Technol 40:5629-5635

3. Löffler D, Ternes TA (2003) Determination of acidic pharmaceuticals, antibiotics and ivermectin in river sediment using liquid chromatography-tandem mass spectrometry. J Chromatogr A 1021: 133-144

4. Ferrer I, Hennion M-C, Barceló D (1997) Immunosorbents coupled on-line with liquid chromatography/atmospheric pressure chemical ionization/mass spectrometry for the part per trillion level determination of pesticides in sediments and natural waters using low preconcentration volumes. Anal Chem 69:4508-4514

5. Reddy CM, Quinn JG, King JW (2000) Free and bound benzotriazoles in marine and freshwater sediments. Environ Sci Technol 34:973-979

6. Schwarzenbach RP, Escher BI, Fenner K, Hofstetter TB, Johnson CA, von Gunten U, Wehrli B (2006) The challenge of micropollutants in aquatic systems. Science 313:1072-1077

7. Giger W (2002) Dealing with risk factors. Eawag News 53e:3-5

8. Krauss M, Singer H, Hollender J (2010) LC-high resolution MS in environmental analysis: from target screening to the identification of unknowns. Anal Bioanal Chem 397:943-951

9. Hernández F, Sancho JV, Ibáñez M, Abad E, Portolés T, Mattioli L (2012) Current use of high-resolution mass spectrometry in the environmental sciences. Anal Bioanal Chem 403:1251-1264

10. Ibáñez M, Sancho JV, Hernández F, McMillan D, Rao R (2008) Rapid non-target screening of organic pollutants in water by ultraperformance liquid chromatography coupled to time-of-light mass spectrometry. TrAC Trends Anal Chem 27:481-489

11. Hogenboom AC, van Leerdam JA, de Voogt P (2009) Accurate mass screening and identification of emerging contaminants in environmental samples by liquid chromatography-hybrid linear ion trap Orbitrap mass spectrometry. J Chromatogr A 1216:510-519

12. Nurmi J, Pellinen J, Rantalainen A-L (2012) Critical evaluation of screening techniques for emerging environmental contaminants based on accurate mass measurements with time-of-flight mass spectrometry. J Mass Spectrom 47:303-312

13. Bueno MJM, Agüera A, Hernando MD, Gómez MJ, Fernández-Alba AR (2009) Evaluation of various liquid chromatography-quadrupolelinear ion trap-mass spectrometry operation modes applied to the analysis of organic pollutants in wastewaters. J Chromatogr A 1216:5995-6002

14. Bobeldijk I, Vissers JPC, Kearney G, Major H, van Leerdam JA (2001) Screening and identification of unknown contaminants in water with liquid chromatography and quadrupole-orthogonal acceleration-time-of-flight tandem mass spectrometry. J Chromatogr A 929:63-74
15. Schymanski EL, Singer HP, Longrée P, Loos M, Ruff M, Stravs MA, Ripollés Vidal C, Hollender J (2014) Strategies to characterize polar organic contamination in wastewater: exploring the capability of high resolution mass spectrometry. Environ Sci Technol 48:1811-1818

16. Moschet C, Piazzoli A, Singer H, Hollender J (2013) Alleviating the reference standard dilemma using a systematic exact mass suspect screening approach with liquid chromatography-high resolution mass spectrometry. Anal Chem 85:10312-10320

17. Martínez Bueno MJ, Agüera A, Gómez MJ, Hernando MD, GarcíaReyes JF, Fernández-Alba AR (2007) Application of liquid chromatography/quadrupole-linear ion trap mass spectrometry and time-of-flight mass spectrometry to the determination of pharmaceuticals and related contaminants in wastewater. Anal Chem 79:93729384

18. Thurman EM, Ferrer I, Zweigenbaum JA, García-Reyes JF, Woodman M, Fernández-Alba AR (2005) Discovering metabolites of post-harvest fungicides in citrus with liquid chromatography/timeof-flight mass spectrometry and ion trap tandem mass spectrometry. $\mathrm{J}$ Chromatogr A 1082:71-80

19. HighChem (2014) Mass frontier, version 6.0. HighChem Ltd./ Thermo Fisher Scientific, Bratislava

20. ACD (2013) MS fragmenter. Advanced Chemistry Development Inc., Toronto

21. Wolf S, Schmidt S, Müller-Hannemann M, Neumann S (2010) In silico fragmentation for computer assisted identification of metabolite mass spectra. BMC Bioinform 11:148

22. Kanehisa M, Goto S, Sato Y, Kawashima M, Furumichi M, Tanabe M (2014) Data, information, knowledge and principle: back to metabolism in KEGG. Nucleic Acids Res 42:199-205

23. NCBI (2014) PubChem http://pubchem.ncbi.nlm.nih.gov/, National Center for Biotechnology Information, USA. Accessed 21 Mar 2014

24. RSC (2014) ChemSpider http://www.chemspider.com/, Royal Society of Chemistry, UK. Accessed 21 Mar 2014

25. Allen F, Greiner R, Wishart D (2014) Competitive fragmentation modeling of ESI-MS/MS spectra for putative metabolite identification. Metabolomics. doi:10.1007/s11306-014-0676-4

26. Horai H, Arita M, Kanaya S, Nihei Y, Ikeda T, Suwa K, Ojima Y, Tanaka K, Tanaka S, Aoshima K, Oda Y, Kakazu Y, Kusano M, Tohge T, Matsuda F, Sawada Y, Hirai MY, Nakanishi H, Ikeda K, Akimoto N, Maoka T, Takahashi H, Ara T, Sakurai N, Suzuki H, Shibata D, Neumann S, Iida T, Funatsu K, Matsuura F, Soga T, Taguchi R, Saito K, Nishioka T (2010) MassBank: a public repository for sharing mass spectral data for life sciences. J Mass Spectrom 45:703-714

27. HighChem (2014) mzCloud https://mzcloud.org, HighChem Ltd., Bratislava, Slovakia. Accessed 31 Mar 2014

28. Smith CA, O'Maille G, Want EJ, Qin C, Trauger SA, Brandon TR, Custodio DE, Abagyan R, Siuzdak G (2005) METLIN: a metabolite mass spectral database. Ther Drug Monitor 26(6):747-751

29. Schymanski EL, Meringer M, Brack W (2011) Automated strategies to identify compounds on the basis of GC/EI-MS and calculated properties. Anal Chem 83:903-912

30. Virtual Computational Chemistry Laboratory (VCCLAB) (2005) http://www.vcclab.org. Accessed 9 Mar 2014

31. Kern S, Fenner K, Singer HP, Schwarzenbach RP, Hollender J (2009) Identification of transformation products of organic contaminants in natural waters by computer-aided prediction and high-resolution mass spectrometry. Environ Sci Technol 43:7039-7046

32. Loos M, Ruff M, Singer H. enviMass version 1.2 target screening software, http://www.eawag.ch/forschung/uchem/software/ enviMass1 2. Accessed 9 Mar 2014

33. Van Leeuwen SPJ, de Boer J (2007) Extraction and clean-up strategies for the analysis of poly- and perfluoroalkyl substances in environmental and human matrices. J Chromatogr A 1153:172-185

34. Liechti P, Der Zustand der Seen in der Schweiz (1994) Bundesamt für Umwelt, Wald und Landschaft BUWAL: Bern 
35. Little J, Cleven C, Brown S (2011) Identification of "known unknowns" utilizing accurate mass data and chemical abstracts service databases. J Am Soc Mass Spectrom 22:348-359

36. Beller HR, Simoneit BRT (1988) Hexachlorophene distributions in estuarine sediments. Bull Environ Contam Toxicol 41:645-650

37. Antonic J, Heath E (2007) Determination of NSAIDs in river sediment samples. Anal Bioanal Chem 387:1337-1342

38. Cole RB, Zhu J (1999) Chloride anion attachment in negative ion electrospray ionization mass spectrometry. Rapid Commun Mass Spectrom 13:607-611

39. Mathis JA, McCord BR (2005) The analysis of high explosives by liquid chromatography/electrospray ionization mass spectrometry: multiplexed detection of negative ion adducts. Rapid Commun Mass Spectrom 19:99-104

40. Zhu J, Hou Y, Feng Y-1, Shoeib M, Harner T (2008) Identification and determination of hexachlorocyclopentadienyldibromocyclooctane (HCDBCO) in residential indoor air and dust: a previously unreported halogenated flame retardant in the environment. Environ Sci Technol 42:386-391

41. EnviPat Web 1.3 (2013) Isotopic pattern and profile calculator. Eawag, Dübendorf http://www.envipat.eawag.ch/index.php

42. Tomlin CDS (1995) The pesticide manual, 10th edn. Royal Society of Chemistry, Cambridge, and British Crop Protection Council, London 